\title{
Diagnostic imaging for hepatocellular carcinoma
}

\author{
Adriano de Santis, Giulia Gallusi \\ Department of translational and precision Medicine, Sapienza University of Rome, Rome 00185, Italy.
}

Correspondence to: Dr.Adriano de Santis, Department of translational and precision Medicine, Sapienza University of Rome, Rome 00185, Italy. E-mail: adsdmc@tin.it How to cite this article: de Santis A, Gallusi G. Diagnostic imaging for hepatocellular carcinoma. Hepatoma Res 2019;5:1.
http://dx.doi.org/10.20517/2394-5079.2018.65

Received: 25 May 2018 First Decision: 1 Aug 2018 Revised: 12 Dec 2018 Accepted: 13 Dec 2018 Published: 8 Jan 2019

Science Editor: Guang-Wen Cao Copy Editor: Cui Yu Production Editor: Huang-Liang Wu

\begin{abstract}
Hepatocellular carcinoma (HCC) occurs mostly in individuals with cirrhosis, which is why the guidelines of the most important scientific societies indicate that these patients are included in surveillance programs through the repetition of an ultrasound examination every 6 months. The aim is to achieve early identification of the neoplasia in order to increase the possibility of curative therapies (liver transplantation, surgery or local ablative therapies) and to increase patient survival. HCC nodules arising in cirrhotic livers show characteristic angiographic behavior that can be evaluated with dynamic multidetector computed tomography and dynamic magnetic resonance imaging (MRI). However, the use of these techniques in real life is often hindered by the lack of uniform terminology in reporting and in the interpretation of the exams reflected in the impossibility of comparing examinations performed in different centers and/or at different times. Liver Imaging Reporting and Data System ${ }^{\circledR}$ was created to standardize reporting and data collection of computed tomography and MRI for HCC. In some cases HCC arises in patients with healthy livers and, although there is evidence that angiographic behavior is not different from cirrhotic patients in this clinical situation, the guidelines still indicate the execution of a biopsy. Frequent use of palliative therapeutic techniques such as transarterial chemoembolization, transarterial radioembolization or administration of antiangiogenic drugs (sorafenib) poses problems of interpretation of the therapeutic response with repercussions on the subsequent choices that have been attempted to resolve with the use of stringent criteria such as Modified Response Evaluation Criteria In Solid Tumors.
\end{abstract}

Keywords: Hepatocellular carcinoma, cirrhosis, ultrasonography, magnetic resonance imaging, multidetector computed tomography, Liver Imaging Reporting and Data System, Modified Response Evaluation Criteria In Solid Tumors

\section{INTRODUCTION}

The diagnosis of hepatocellular carcinoma (HCC) can be addressed in two different clinical settings. A first context, rarely, is that of the patient with a healthy liver. In this scenario, patients do not undergo routine 
monitoring and tumors are often large with possible vascular involvement. In these patients, performing a liver biopsy is often necessary for diagnostic confirmation. A second, frequently, is that of patients with chronic liver disease (cirrhosis or advanced fibrosis) under regular ultrasound surveillance ${ }^{[1]}$. The goal of surveillance and screening is to reduce mortality ${ }^{[2]}$. For the aforementioned reasons HCC meets the criteria for developing a surveillance program and the use of ultrasound as a screening tool is accepted by major scientific societies $^{[3-5]}$. In many studies the 3 -year survival rate varies between $50.8 \%$ and $45.6 \%$ in patients under surveillance and $27.9 \%$ and $28.8 \%$ in those not screened. Even after correction for lead time bias, the three-year survival is better in patients undergoing ultrasound surveillance: $39.7 \%$ vs. $29.1 \%$. It is likely that the increased survival in patients undergoing surveillance is linked to an increase in early detection of HCC in patients screened (OR of 2.11; 95\% CI, 1.88-2.33) and therefore of the use of curative treatments $(61.8 \% \text { vs. } 38.2 \%)^{[5]}$.

\section{ULTRASONOGRAPHY AND CONTRAST-ENHANCED ULTRASONOGRAPHY}

Efficacy of detection of HCC with ultrasound varies widely and in cirrhotic patients presents with a sensitivity of $33 \%-96 \%{ }^{[6]}$ while specificity reach over $90 \%{ }^{[7]}$. The identification of small HCC nodules in cirrhotic liver with a coarse parenchymal pattern is not easy, therefore a skilled operator must work with adequate equipment, preferably in dedicated centers. In gray-scale ultrasound small HCCs typically appear as a hypoechoic lesion. In some cases increased echogenicity may be present due to adipose degeneration. Sometimes the hypoechoic nodule may present a hyperechoic focus which is suggestive of development of HCC within a dysplastic nodule (nodule in nodule phenomenon) ${ }^{[8]}$.

But not all nodules identified with ultrasound in patients with chronic liver disease (cirrhosis or advanced fibrosis) undergoing surveillance are HCCs. In this context, the role of imaging is to differentiate the nodules of HCC from other malignant lesions (intrahepatic cholangiocarcinoma and metastasis) and nonmalignant (e.g., regenerative nodules, low and high grade dysplastic nodules, confluent fibrosis, angiomas, etc.) that can be found in the cirrhotic liver. In oncology, the diagnosis of cancer generally requires histological assessment; from this point of view HCC is an exception since a non-invasive diagnosis can be achieved with imaging alone in these high-risk populations. The peculiar angiographic behavior of the HCC nodules in a cirrhotic liver characterized by the presence of the wash-in during the arterial phase and by the wash-out during the venous and late phases, represents the diagnostic hallmarks of HCC. These characteristics are able to provide a reliable diagnosis of HCC in high-risk patients affected by liver cirrhosis or with advanced fibrosis, and represent the background for the development, by Western and Asian scientific societies, of different algorithms for non-invasive diagnosis of $\mathrm{HCC}^{[3,4,9,10]}$. The recommended imaging methods are computed tomography (CT) and MRI with contrast agents. Diagnostic algorithms of American Association for the Study of Liver Diseases (AASLD) and European Association for the Study of the Liver (EASL) guidelines adopt a strategy dependent on the size of the lesion. Nodules smaller than 1 $\mathrm{cm}$ are considered too small to be characterized and both guidelines recommend ultrasound monitoring every three (AASLD) or four months (EASL). As for the diagnosis of nodules with a diameter greater than $2 \mathrm{~cm}$, both guidelines recommend only one imaging method. For nodules with a diameter of $1-2 \mathrm{~cm}$, the statements differ, as the American guidelines recommend the same approach used for lesions larger than $2 \mathrm{~cm}$ (only one contrast method is sufficient), while the European guidelines contemplate the concordance of two consecutive images if these cases are not followed in centers with substantial "expertise".

Consequently, in cirrhotic patients, biopsy is indicated only in cases where nodules do not present contrasting features typical of HCC. However, the increasing knowledge about the immunohistochemical and molecular characteristics of HCC may bring biopsy to the forefront in order to select patients who could gain the most benefit from target-driven HCC treatments ${ }^{[11]}$.

In conclusion, the identification of a liver focal lesion greater than $1 \mathrm{~cm}$ in the course of surveillance of patients at risk with ultrasonography imposes the study of the nodule vascularization through the use of 
multidetector computed tomography (MDCT) or multi-phase nuclear magnetic resonance imaging (MRI) with contrast agents.

Contrast-enhanced ultrasound (CEUS) may be a useful imaging modality for the noninvasive diagnosis of small, newly detected liver nodules during surveillance of cirrhotic patients ${ }^{[12]}$. Ultrasound contrast agents ("microbubbles") comprise an albumen or phospholipid shell containing a stable perfluorocarbon or sulphur hexafluoride gas. They are predominantly blood-pool agents, as the encapsulated microbubbles are small enough to pass through both pulmonary and systemic circulation after intravenous injection and durable enough to re-circulate for several minutes ${ }^{[13]}$. CEUS can also be utilized in the presence of renal impairment and can be performed at the time in which the lesion is discovered but it does not eliminate the need for CT and/or MRI in order to characterize the lesion and to stage the disease ${ }^{[14]}$. CEUS was inserted as a method for characterizing nodules arising in cirrhotic livers, in the 2005 AASLD guidelines ${ }^{[15]}$ but was subsequently eliminated in 2011, partly due to lack of availability of ultrasound contrast in the USA and partly due to false positive diagnoses in patients with intra-hepatic cholangiocarcinoma ${ }^{[3]}$.

\section{CT AND MRI}

As already mentioned, the identification of a liver focal lesion greater than $1 \mathrm{~cm}$ in the course of surveillance with ultrasonography of patients at risk imposes the study with higher level image techniques such as MDCT or MRI with an extracellular contrast medium (iodized compound or gadolinium-based compounds: gadoteric acid, gadopentetic acid, gadodiamide, gadoteridol, gadobutrol) that remain in the extracellular space and allows the characterization of blood flow. Multi-phase MRI may be performed also with an hepatospecific contrast agents such as gadolinium ethoxybenzyl diethylenetriamine pentaacetic acid or Gd-EOB-DTPA, gadobenate dimeglumine or Gd-BOPTA, which is captured by "healthy" hepatocytes and excreted in the biliary tract, or by iron oxide particles (SPIO) with superparamagnetic activity, which are captured by Kupffer cells located in the non-neoplastic hepatic parenchyma and in benign lesions but not in malignant ones.

The goals of the evaluation by MRI or MDCT of a hepatic nodule in a patient with liver cirrhosis are not only the determination of the nature of the lesion but also, in the case of an HCC, the estimate of the hepatic extension of the neoplasia and the possible localization in extrahepatic sites in order to propose a treatment based on the exact staging of the disease.

There is universal consensus that the diagnosis of HCC can be achieved without biopsy in a situation where the pre-test probability is very high, as happens in liver cirrhosis, but there is no consensus as to which technique is the best. The angiographic features of HCC are identical in MDCT and MRI, but the latter offers a series of additional imaging sequences such as T2-weighted sequences, diffusion-weighted imaging and in combination with the use of a hepatospecific contrast agent it can improve diagnostic performance ${ }^{[16-19]}$. However, MRI presents greater technical complexity, longer scan times, greater susceptibility to artifacts, a less consistent image quality, higher cost, lower availability, longer scheduling backlogs ${ }^{[20]}$ and its diagnostic yield becomes void if the patient is unable to hold his breath, to remain still or presents a high-volume ascites. For these reasons the superiority of one method over the other, especially in real-life contexts remains uncertain.

In a recent meta-analysis in which MDCT was compared with MRI with an extracellular agent, or MRI with gadoxetate disodium, Roberts et al. ${ }^{[20]}$ concluded that the latter showed significantly higher sensitivity (0.82; 95\% CI, 0.75-0.87 vs. 0.66; $95 \%$ CI, 0.60-0.72) and lower negative likelihood ratio (0.20; 95\% CI, 0.15-0.28; vs. 0.37; 95\% CI, 0.30-0.44) in diagnosis of HCC lesions. Pooled analysis demonstrated that both gadoxetate enhanced MRI and extracellular contrast - enhanced MRI provided significantly higher sensitivity and 
lower negative likelihood ratio than $\mathrm{MDCT}^{[20]}$. However, the authors do not believe there is enough evidence to provide definitive recommendation for systematic use of gadoxetate-enhanced MRI or extracellular contrast-enhanced MRI over MDCT. In fact, in clinical practice, beyond the diagnostic yield, many other factors may guide the choice between modalities, such as the presence of ascites, the patient's inability to hold his or her breath, the severity of cirrhosis and/or a significant hepatic iron overload, and the presence of contraindications to the use of contrast agents.

\section{LIVER IMAGING REPORTING AND DATA SYSTEM}

The application of the guidelines in real life is often penalized by the lack of uniform terminology in reporting and by the excessive variation in the interpretation of the exams causing the impossibility of comparing examinations performed in different centers and/or at different times. Liver Imaging Reporting and Data System ${ }^{\odot}\left(\text { LI-RADS }^{\circledR}\right)^{[21]}$ was created to standardize the reporting and data collection of MDCT and MRI for HCC. This method of categorizing liver findings for patients with risk factors for developing HCC allows the radiology community to: (1) apply consistent terminology; (2) reduce imaging interpretation variability and errors; (3) Enhance communication with referring clinicians; and (4) facilitate quality assurance and research.

LI-RADS, was originally released by the American College of Radiology in 2011, and since then revised four times. The system was created to be applied to MDCT and MRI in the context of hepatic diseases at high risk of developing malignant lesions, such as cirrhosis, chronic hepatitis B or history of current or prior HCC. They permit better communication between radiologists and physicians, clearly differentiating between lesions definitively benign (LR1, i.e., LI-RADS 1), probably benign (LR2), with intermediate probability of being malignant (LR3), with high probability of being malignant but not necessarily HCC (LR-M), probably HCC (LR4), and definitively HCC (LR5). The final version of LI-RADS has been published online on the American College of Radiology (ACR) website ${ }^{[22]}$. The assignment to specific categories is obtained considering certain "major features": (1) arterial phase hyperenhancement; (2) size of the lesion; (3) portal venous phase wash-out; (4) "enhancing capsule" in portal venous/delayed/transitional phases; and (5) speed of growth over a threshold. The "enhancing capsule" is a smooth, uniform, sharp border around the lesion, clearly thicker than the fibrous layers of the background regenerative cirrhotic nodules. The threshold of growth means an increase in size of a mass by a minimum of $5 \mathrm{~mm}$ associated with: $\geq 50 \%$ increase in size in $\leq 6$ months, $\geq 100 \%$ increase in size in $>6$ months or a previously unseen nodule on MDCT/MRI, now $\geq 10 \mathrm{~mm}$, in $\leq 24$ months. These "major criteria" must be combined as shown below to ascertain the final category [Figure 1]. The use of "ancillary criteria" is at the discretion of the radiologist, and allows for recategorization [Figure 2]. In fact, one ancillary feature favoring malignancy, allows for upgrading by one category to LR-4 (but can never be used to upgrade to LR-5); on the contrary, one ancillary criterion favoring benignity warrants downgrading by one category; the coexistence of one criterion favoring benignity and another favoring malignity does not modify the current category.

LI-RADS are also applicable in judging the response to treatment: even if there is no description of treatment-specific features, some general indications are given to carry out the categorization, as illustrated in Table 1.

Regarding the category "LR-TR viable", when the tissue has a thick irregular aspect, the measurement is made by taking the longest diameter of the enhancing area, without traversing the non-enhancing area; when it has a mass like aspect (and possibly more than one mass), the biggest enhancing area is to be measured, by taking its longest diameter.

Compared to other systems for radiological evaluation of hepatic lesions, LI-RADS has introduced an important innovation that is a program of follow-up for each radiological category. Specifically, benign 


\section{CT/MRI Diagnostic Table}

\begin{tabular}{|c|c|c|c|c|c|c|}
\hline \multirow{2}{*}{\multicolumn{2}{|c|}{$\begin{array}{l}\text { Arterial phase hyperenhancement (APHE) } \\
\text { Observation size }(\mathrm{mm})\end{array}$}} & \multicolumn{2}{|c|}{ No APHE } & \multicolumn{3}{|c|}{ APHE (not rim) } \\
\hline & & $<20$ & $\geq 20$ & $<10$ & $10-19$ & $\geq 20$ \\
\hline \multirow{3}{*}{$\begin{array}{l}\text { Count major features: } \\
\text { - "Washout" (not peripheral) } \\
\text { - Enhancing "capsule" } \\
\text { - Threshold growth }\end{array}$} & None & LR-3 & LR-3 & LR-3 & LR-3 & LR-4 \\
\hline & One & LR-3 & LR-4 & LR-4 & $R-4$ & LR-5 \\
\hline & $\geq$ Two & LR-4 & LR-4 & LR-4 & LR-5 & LR-5 \\
\hline
\end{tabular}

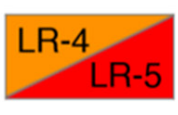

Observations in this cell are categorized LR-4, except:

- LR-5g, if $\geq 50 \%$ diameter increase in $<6$ months (equivalent to OPTN 5A-g)

- LR-5us, if "washout" and visibility at screening ultrasound (per AASLD HCC criteria)

\section{If unsure about the presence of any major feature: characterize that feature as absent}

Figure 1. Categories Liver Imaging Reporting and Data System based on the application of major criteria. CT: computed tomography; MRI: magnetic resonance imaging; LR: Liver Imaging Reporting and Data System (LI-RADS); APHE: arterial phase hyperenhancement; OPTN: Organ Procurement and Transplantation Network; AASLD: American Association for the Study of Liver Diseases; HCC: hepatocellular carcinoma

Table 1. Liver Imaging Reporting and Data System criteria to define the response to treatment

\begin{tabular}{ll}
\hline Responsecategory & Criteria \\
\hline LR-TR nonviable & No lesional enhancement OR \\
& Treatment-specific expected enhancement pattern \\
LR-TR equivocal & Enhancement atypical for treatment-specific expected enhancement pattern and not meeting criteria for \\
& probably or definitely viable \\
LR-TR viable & Nodular, masslike, or thick irregular tissue in or along the treated lesion with any of the following: \\
& Arterial phase hyperenhancement OR \\
& Washout appearance OR \\
& Enhancement similar to pretreatment \\
\hline
\end{tabular}

LR-TR: Liver Imaging Reporting and Data System Treatment Response

lesions (LR-1 and LR-2) do not require an adaptation of the normal program of surveillance proposed by the guidelines for patients at risk of HCC, so in this condition a MDCT/MRI with extracellular contrast should be repeated after 6 months. For lesions at intermediate risk of malignancy (LR-3), it is advised to repeat the same imaging examination at 3-6 months; changing the imaging technique is a possible alternative, but is not recommended. For LR-M and LR-4, a multisciplinary discussion is required to decide whether a biopsy and/or treatment is feasible, otherwise the same imaging examination will be repeated within a maximum of 3 months. A multidisciplinary discussion is also proposed for LR-5 to select the best treatment option. A special category that needs multidisciplinary evaluation is that of LR-TIV (tumor in vein) which is assigned only if the neoplastic nature of the vascular occlusion can unequivocally be determined, combining radiological features with serological biomarkers and (if needed) histological aspect. For treated HCC, independently from the result obtained, a follow-up every 3 months or less using the same imaging modality is suggested. Reporting this last recommendation in clinical practice, a reasonable approach could be that of monitoring the treated lesion with ultrasound as well, in order to better guide the timing for the repetition of MDCT/MRI on the basis of dimensional or aspect modification of HCC.

As mentioned before, ultrasound is the screening method indicated by all guidelines for the surveillance of patients at risk of developing an HCC. In light of the potential importance of a first detection in ultrasound 
Ancillary features favoring malignancy

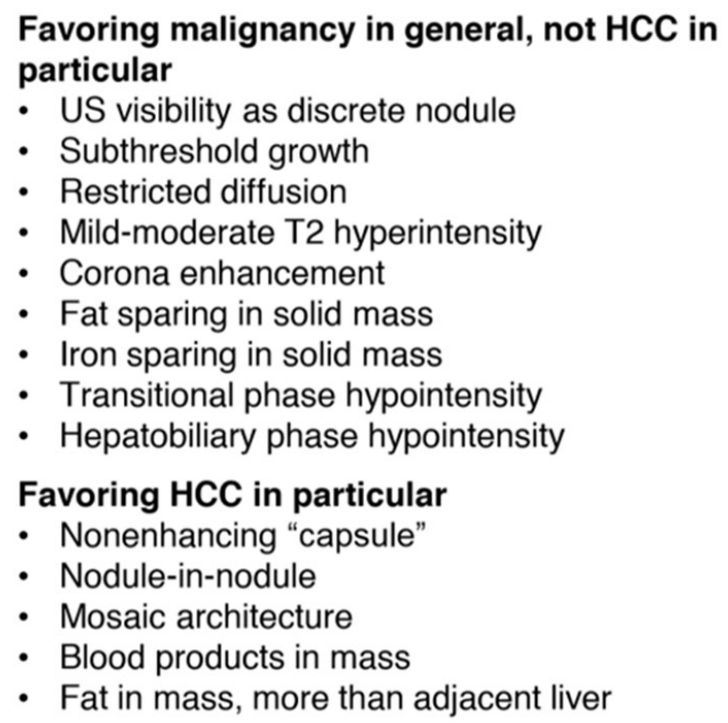

\section{If unsure about presence of any ancillary feature: characterize that feature as absent}

Figure 2. Ancillary features can help the radiologist to upgrade or downgrade of category the hepatic lesions. US: ultrasound; HCC: hepatocellular carcinoma

of nodules suspected of being malignant, ACR has proposed LI-RADS ultrasound v2017 ${ }^{[23]}$ criteria for the definition of the risk on the basis of the features depicted at the radiological examination of the liver by ultrasound. The first step is to determine the presence/absence of a focal lesion and to subsequently identify the appropriate LI-RADS category: "US-1" (negative) defines the absence of lesions or the presence of clearly benign observations such as cysts, hemangiomas or skip areas around the gallbladder fossa; "US-2" (subthreshold) refers to a solid nodule which is not unequivocally benign, of diameter $\leq 1 \mathrm{~cm}$ and warrants short-term ultrasound surveillance; "US-3" (positive) takes into account lesions $\geq 10 \mathrm{~mm}$ in diameter, not unequivocally benign, which may warrant multiphase contrast enhanced imaging. This latter category also comprises new venous thrombosis. Considering the possible limitations of visibility at ultrasound associated with technical difficulties such as large patient body habitus or inability to cooperate, limited acoustic window, parenchymal heterogeneity and/or reduced beam penetration, LI-RADS ultrasound allows for the use of a "visualization score": (1) no or minimal limitations which are unlikely to meaningfully affect sensitivity; (2) moderate limitations which may obscure small masses; and (3) severe limitations which significantly lower sensitivity for focal liver lesions. The category US-1 requires continuation of screening/ surveillance with ultrasound every six months; US-2 demands follow up by ultrasound after 3-6 months; US-3 warrants immediate multiphase contrast-enhanced MDCT/MRI or CEUS ${ }^{[23]}$.

Contrast agents for ultrasound are biodegradable microbubbles that resonate under low-power ultrasound waves and generate harmonic signals. A contrast-specific ultrasound imaging mode, available on the majority of ultrasound scanners, highlights signals from microbubbles while applying specific pulse sequences which suppress signals from tissues. This stimulation of the microbubbles allows for the visualization of arterial hyper-enhancement and venous wash-out. Prospective studies have added evidence that different hepatic malignant lesions appear differently in CEUS and that their post-contrast behaviour is typical and reproducible ${ }^{[24]}$. As such, ACR has included a section dedicated to CEUS firstly in the version of LI-RADS of 2016 and has recently published a new edition ${ }^{[25,26]}$. Similar to those for injected MDCT/MRI, LI-RADS categories for CEUS are: CEUS LR-NC (uncategorizable), CEUS LR-TIV, CEUS LR-1 (definitely 


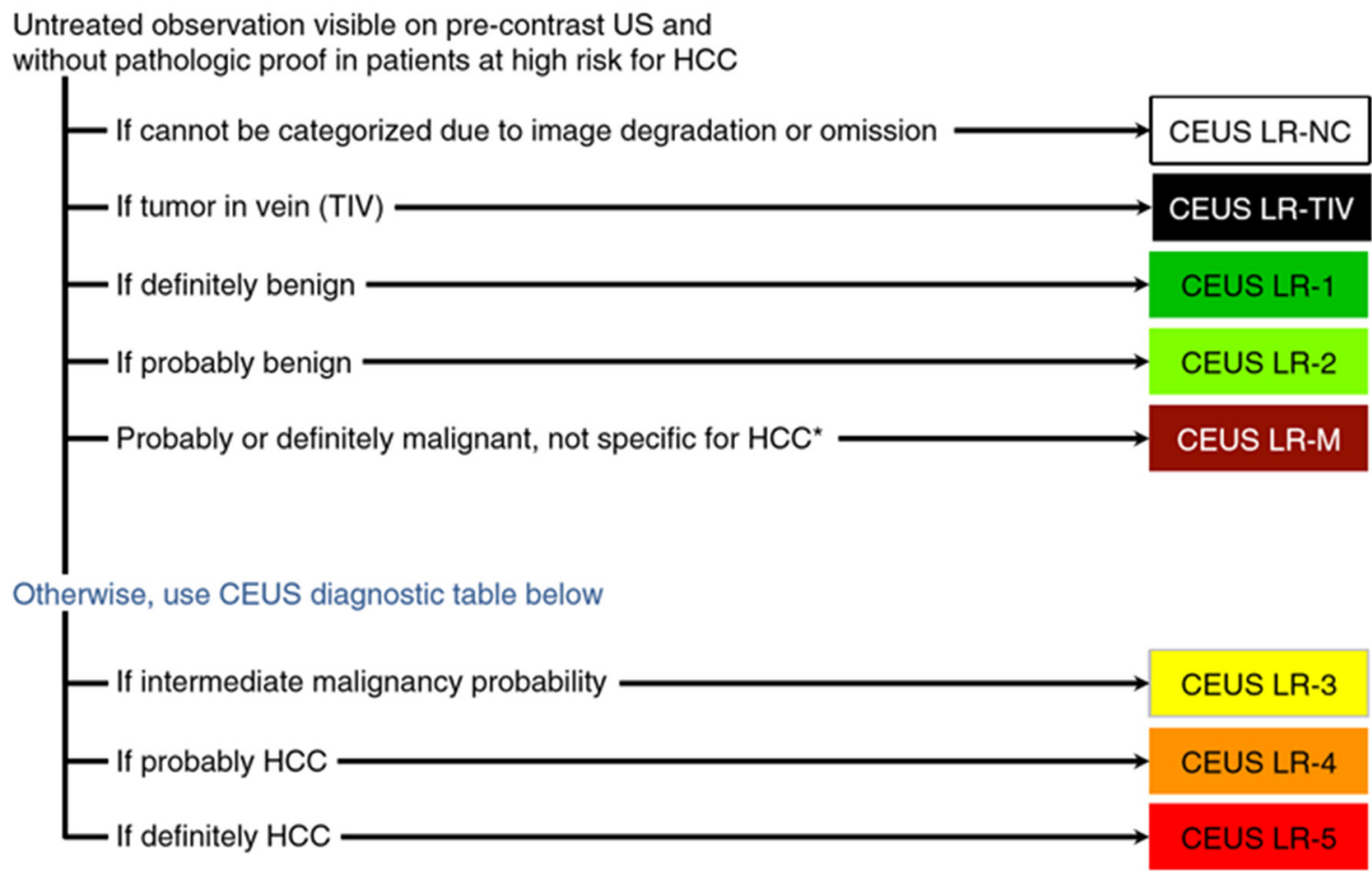

\section{CEUS Diagnostic Table}

\begin{tabular}{|c|c|c|c|c|}
\hline $\begin{array}{l}\text { Arterial phase hyperenhancement } \\
\text { (APHE) }\end{array}$ & & APHE & & \\
\hline Nodule size $(\mathrm{mm})$ & $<20$ & $\geq 20$ & $<10$ & $\geq 10$ \\
\hline No washout of any type & CEUS LR-3 & CEUS LR-3 & CEUS LR-3 & CEUS LR-4 \\
\hline Late and mild washout & CEUS LR-3 & CEUS LR-4 & CEUS LR-4 & CEUS LR-5 \\
\hline $\begin{array}{r}{ }^{*} \text { CEUS LR-M criteri } \\
\text { If rim APHE OR } \\
\text { early }(<60 \text { s) was } \\
\text { marked washou }\end{array}$ & & $\begin{array}{l}\text { APHE: } \\
\text { Not rim (indic } \\
\text { Not periphera } \\
\text { hemangioma }\end{array}$ & $\begin{array}{l}\text { LR-M) } \\
\text { continuous g }\end{array}$ & r (indicates \\
\hline
\end{tabular}

Figure 3. Contrast-enhanced ultrasound Liver Imaging Reporting and Data System. LR: Liver Imaging Reporting and Data System (LIRADS); US: ultrasound; CEUS: contrast-enhanced ultrasound; LR-NC: LI-RADS noncategorizable; TIV: tumor in vein; HCC: hepatocellular carcinoma; APHE: arterial phase hyperenhancement; LR-M: LI-RADS malignancy

benign), CEUS LR-2 (probably benign), CEUS LR-M (probably or definitely malignant but not specific for HCC), CEUS LR-3 (with intermediate risk of being malignant), CEUS LR-4 (probably HCC) and CEUS LR-5 (definitely HCC). The designation of categories CEUS LR-NC, LR-TIV, LR-1, LR-2, LR-M is possible in light of a basal observation of the lesion by ultrasound, whereas the designation of CEUS LR-3, LR-4, LR-5 requires a post-contrast study [Figure 3].

Globally, the post-contrast behaviour of hepatic nodules in CEUS does not greatly differ from that observed in MDCT/MRI in terms of arterial hyperenhancement and venous/delayed washout. However, CEUS offers the possibility of studying the region of interest from a closer point of view and, by temporally monitoring the features of enhancement and of wash-out, it is possible to deduce additional and, sometimes, more 


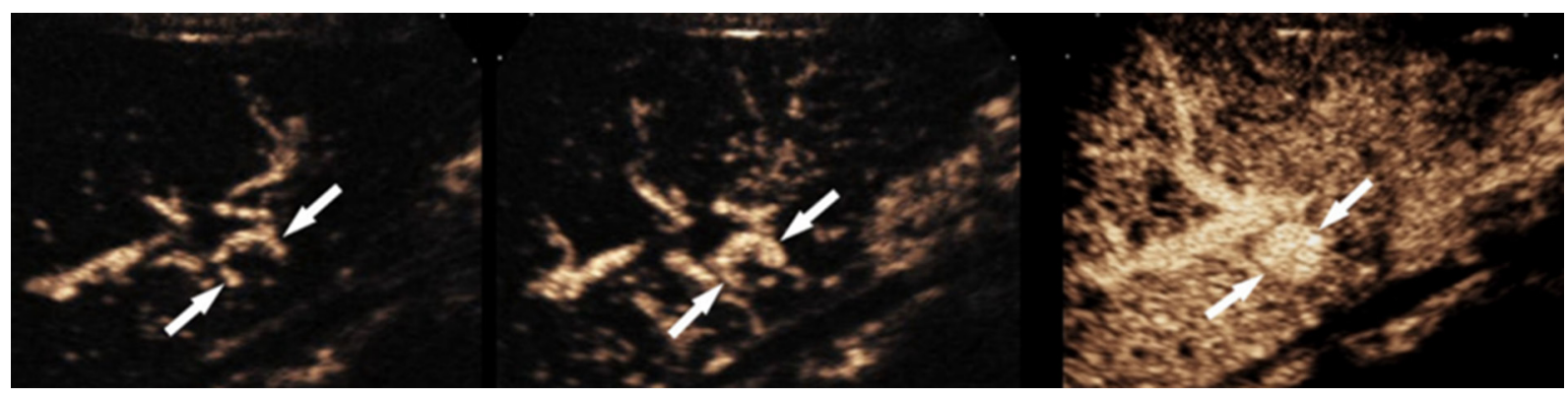

Figure 4. The globular-like progressive centripetal arterial filling of hemangioma

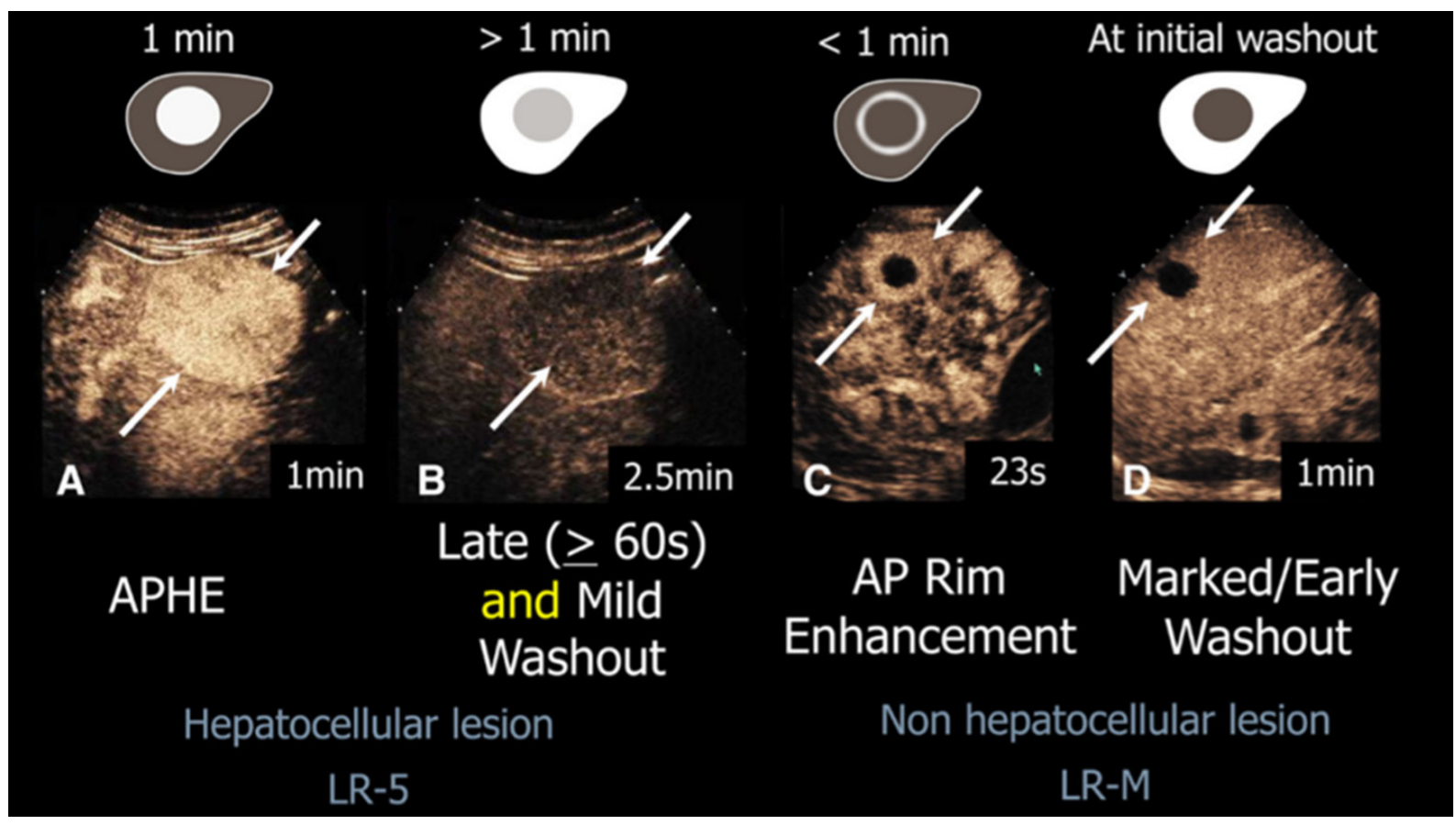

Figure 5. In $A$ and $B$, an $\mathrm{HCC}$ presents arterial hyperenhancement and late and mild washout with respect to the surrounding liver; on the contrary, in $C$ and $D$, a malignant lesion of probable metastatic nature shows rim hyperenhancement together with early and marked wash-out. HCC: hepatocellular carcinoma; APHE: arterial phase hyperenhancement; LR: Liver Imaging Reporting and Data System (LIRADS); LR-M: LI-RADS malignancy

precise information with respect to MDCT/MRI. Obviously, as a counterpart, the global vision of the entire abdomen is lost, such that CEUS is not appropriate for tumor staging. In return, it can clearly depict key details such as the progressively centripetal and globular arterial hyperenhancement typical of hemangiomas (CEUS LR-1) [Figure 4], or the peripheral hyperenhanced rim visible in the early venous phase which is diagnostic of intrahepatic cholangiocarcinoma (CEUS LR-M), the early $(<60 \mathrm{~s})$ and complete wash-out typical of metastases or of intrahepatic cholagiocarcinoma (CEUS LR-M) and which unequivocally differs from late (> $60 \mathrm{~s}$ ) and partial wash-out which are diagnostic of HCC (CEUS LR-5) [Figure 5].

These are only some examples given in order to emphasize that CEUS has great potential to help adjudicate the right diagnosis, not only in the event of hepatic nodules of uncertain nature and as a complementary diagnostic tool after MDCT/MRI, but also as a first post-contrast examination of an observation of uncertain nature made at ultrasound (i.e., distinction between real nodule $v$ s. fat sparing area/accumulation). In many situations CEUS can define the real nature of a lesion with high sensibility and specificity and can avoid an unneeded biopsy or, on the contrary, guide in its realization, in the presence of a hepatic 

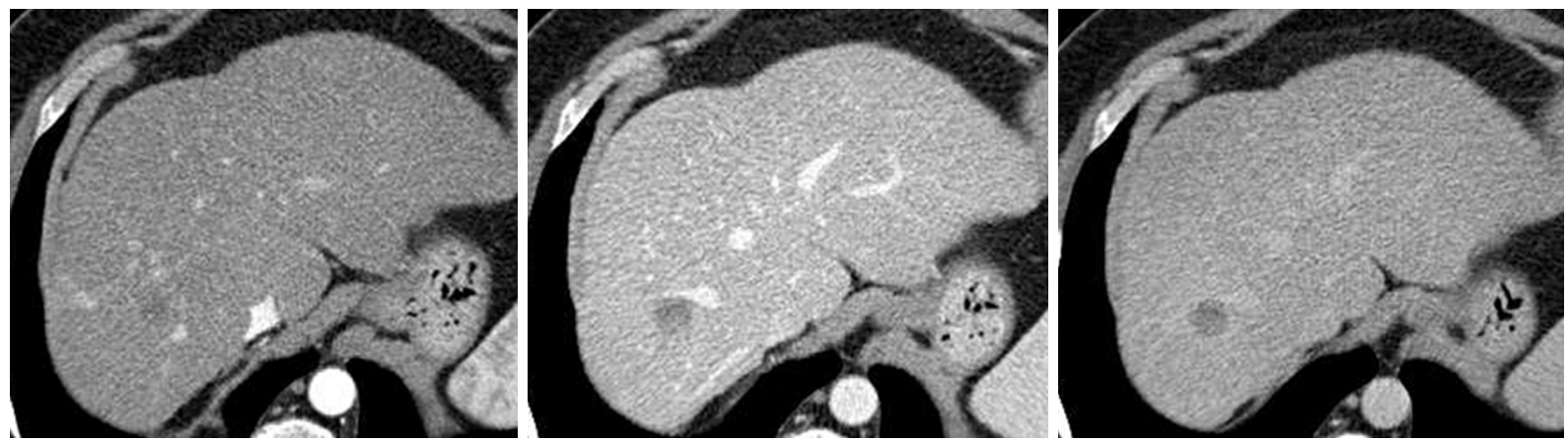

Figure 6. From left to right: hepatic arterial phase, venous and late phase on multidetector computed tomography shows no enhancement of the tumor (due to the courtesy of Dr. Michele Di Martino)

lesion that undoubtedly exists but is scarcely visible with a basal ultrasound evaluation. In a recent study including 1,006 nodules, 820 (81\%) HCC, 40 (4\%) cholangiocarcinoma, 116(11\%) regenerative/dysplastic nodules), Terzi et al. ${ }^{[27]}$ demonstrated that the LR-5 category(52\% of all nodules) was $98.5 \%$ predictive of HCC, with no risk of misdiagnosis for pure cholangiocarcinoma. Sensitivity for HCC was $62 \%$. All LR-M nodules were malignant and the majority was of non-hepatocellular origin. The LR-3 category included 203 lesions [HCC 96 (47\%)] and the LR-4 202 [HCC 173 (87\%)]. These and similar results confirm the utility and the great potential of CEUS and justify the re-introduction of CEUS into guidelines. In the latest version of the EASL guidelines, CEUS was introduced in the diagnostic algorithm of HCC in cirrhotic patients but with a moderate degree of evidence and a weak degree of recommendation ${ }^{[28]}$.

\section{INTERPRETATION OF “NON-HYPERVASCULAR NODULES” IN CIRRHOSIS}

The transformation of a regenerative nodule of cirrhosis into a dysplastic lesion involves a progressively reduced portal venous supply and a progressively increased arterial vascularization with sinusoidal capillarization and recruitment of unpaired arterioles; because of this reduced venous drainage, fat content frequently increases in early HCC but regresses in moderately differentiated HCC. Initially, dysplastic nodules show siderosis and copper retention, while during neoplastic transformation, Kupffer cell density decreases, and iron and copper accumulation are gradually lost ${ }^{[29]}$. Injected MDCT and, even better, MRI, can potentially depict all these changes in a rather sensible way and many efforts toward systematization of imaging description and classification have been made and are still made to promote their correct interpretation. In fact, the systems for radiological assessment of hepatic lesions like LI-RADS are based on the analogy between pathological characteristics and specific radiological features. The main limit of LI-RADS is that a diagnosis of HCC is reached only in the presence of arterial hyperenhancement. Thereby, a hepatic nodule that has a non-hypervascular arterial phase, even in the presence of ancillary features suggestive of malignancy, can never be defined as more than a "probable HCC" (LR-4) [Figure 6] ${ }^{[21]}$.

A study that has evaluated the enhancement pattern at multiphasic MDCT of 204 pathologically proven HCC smaller than $3 \mathrm{~cm}$ in diameter in cirrhotic patients, has found that the predominant enhancement patterns of HCC differ significantly depending on tumor size and cellular differentiation. Up to $46 \%$ of HCCs smaller than $10 \mathrm{~mm}$ in diameter do not show arterial hyperenhancement, while it is found in $70 \%$ of HCCs measuring 10-19 $\mathrm{mm}$ in diameter and in $75 \%$ of those measuring 20-29 $\mathrm{mm}$. In line with these results, the association of arterial hyperenhancement and portal venous washout is observed only in $24 \%$ of $0-9 \mathrm{~mm}$ vs. $28 \%$ of $10-19 \mathrm{~mm}$ vs. $47 \%$ of $20-29 \mathrm{~mm}$ HCCs. Cell differentiation also plays an important role: arterial hyperenhancement is found in only $53 \%$ of well-differentiated HCCs, whereas the prevalence increases to $79 \%$ in moderately differentiated HCCs, and was $60 \%$ in poorly differentiated HCCs. In conclusion, this and similar studies confirm that, although large nodules are easily diagnosed, the main difficulty in imaging of cirrhotic patients is the characterization of hepatic nodules smaller than $2 \mathrm{~cm}$ in diameter as they frequently do not show the "classical" arterial hyperenhancement ${ }^{[30]}$. 

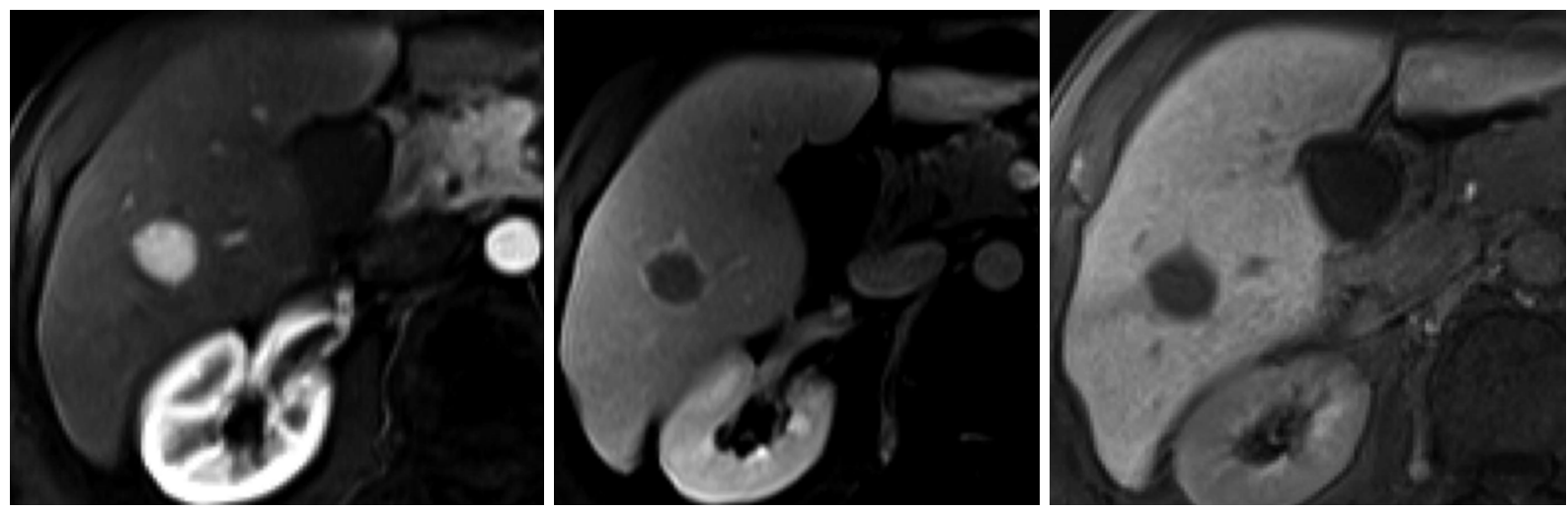

Figure 7. From left to right: hepatic arterial phase of gadoxetic acid-enhanced magnetic resonance imaging shows homogeneous marked enhancement of the tumor; transitional phase shows washout of the contrast medium in the tumor with capsular enhancement; hepatobiliary phase shows marked hypointensity of the tumor relative to the liver parenchyma. Modified from Park et al. ${ }^{[31]}$

In light of these considerations, reaching a definitive non invasive diagnosis of HCC is still a challenge and MDCT and MRI with injection of extracellular agents (ECA) have proven to be relatively useless in the presence of small hypovascular nodules. The commercialization of gadoxetic acid has represented an important step towards the radiological diagnosis of borderline nodules because, it initially distributes in the extracellular fluid compartment, similar to extracellular contrast agents, and is subsequently taken up by functioning hepatocytes and excreted into the bile. Consequently, it provides both the benefits of dynamic imaging and the delayed hepatobiliary phase during which it is actively picked up by the hepatocytes through the organic anion transporting polypeptide (OATP) B1/8 transporter, a protein that is almost always lost in hepatocarcinogenesis [Figure 7]. A study conducted on surgically resected hepatocellular nodules has found a clear correlation between grade of histological de-differentiation, loss of expression of these transporters and appearance in MRI of a hypointensity in the hepatobiliary phase due to the lost capacity of intracellular uptake of the contrast agent, while the surrounding normal parenchyma remains strongly enhanced. Specifically, the authors evaluated 72 HCCs nodules to determine the correlation among the enhancement ratio on gadoxetic acid-enhanced MRI, the histological grade of tumour differentiation and the intensity of immunohistochemical OATP8 expression. They observed that all of the well, moderately and poorly differentiated HCCs showed a significantly decreased enhancement ratio compared with the background liver, with the exception of 6 moderately differentiated HCC which demonstrated a definitively increased enhancement ratio compared with the background liver. All of these nodules with "atypical behavior" showed increased OATP8 expression compared with the surrounding liver, while in all other HCCs a significant reduction in immunohistochemical OATP8 expression proportional to the grade of dedifferentiation was found ${ }^{[32]}$.

These findings, confirmed by other studies, open a new scenario for the non-invasive diagnosis of hypovascular HCCs but the diagnostic role of hepatospecific contrast agents should be endorsed and formalized in international guidelines for radiological diagnosis of HCC, given that the last version of LI-RADS (v2017) still provides a single diagnostic algorithm for multiphase MDCT, MRI with ECA, and MRI with gadoxetate disodium. While initially combined for simplicity, the use of a common algorithm for all three imaging methods has a potentially important drawback: emerging evidence suggests that the assigned categories are modality-dependent, with different modalities assigning different categories to the same observation ${ }^{[33]}$.

\section{THE ROLE OF POSITRON EMISSION TOMOGRAPHY}

The use of PET is still restricted in the field of HCC because of its low sensitivity. In a prospectively conducted study on 99 patients with histologically confirmed HCC who underwent 18F-fluoro-deoxyglucose positron 
emission tomography (FDG PET), none of the 7 patients with small tumors ( $<2 \mathrm{~cm}$ in diameter), 18 of 42 patients (43\%) with tumors $2-5 \mathrm{~cm}$ in diameter, and 32 of 41 patients $(78 \%)$ with tumors larger than $5 \mathrm{~cm}$ had positive findings for all index lesions. The sensibility increased in Barcelona clinic liver cancer staging system advanced stage, metastatic HCC and in patients with high levels of alphafetoprotein. All indexed lesions with positivity in FDG PET correlated with significantly lower survival with respect to patients with negative or partially positive $\mathrm{PET}^{[34]}$. As suggested by these results, other trials have demonstrated that PET positivity correlates with HCC aggressiveness, information which can be used to select, in a non-invasive way, candidates for liver transplantation or major liver resection ${ }^{[35]}$. In a retrospective study conducted on 111 patients with HCC, liver transplantation was performed for 91 of these patients and all underwent PET before the intervention. The tumor recurrence rate after liver transplantation was 3.6\% for patients with non- $[18 \mathrm{~F}]$ FDG-avid PET tumors, but it was $54.3 \%$ for patients with $[18 \mathrm{~F}] \mathrm{FDG}$-avid PET tumors $(P<0.001)$. The 5 -year recurrence-free survival rates were comparable for patients with tumors meeting the Milan criteria (86.2\%) and patients with PET negative HCC exceeding the Milan criteria (81\%) at liver transplantation, but these rates were significantly higher than the rate for liver recipients with [18F]FDG-avid advanced HCC $(21 \%, P<0.002)^{[36]}$.

Preoperative evaluation of HCC with FDG PET has shown that well-differentiated and some moderately differentiated HCCs do not present FDG uptake exceeding that of the surrounding normal liver, whereas poorly differentiated and undifferentiated HCCs have positive PET findings. The standardized uptake value (SUV) max of sarcomatous HCC is much higher than that of poorly differentiated HCC. The entity of FDG captation of both sarcomatous HCC and combined HCC-cholangiocarcinoma is significantly associated with tumor differentiation, tumor size, microvascular invasion and with poor prognosis after surgery ${ }^{[37]}$.

A recently published study conducted on 207 consecutive patients with monofocal HCC undergoing hepatic resection used pre-operative FDG-PET imaging to stratify tumor aggressiveness and the albumin-bilirubin (ALBI) grade to stratify the hepatic reserve. The ALBI grade is a simple and objective measurement of liver function that uses only serum albumin and bilirubin levels and can be applied to all grades of chronic hepatic diseases, unlike Child Pugh score which is restricted to liver cirrhosis. The study demonstrated a strong correlation between the values of ALBI, the ratio tumorSUV/non-tumorSUV (TNR) and endpoints like overall survival and disease-free survival; whereas tumor size and tumor markers were not significant. Moreover, a high pre-operative TNR showed to be significantly associated with extrahepatic recurrence patterns ${ }^{[38]}$.

The role of PET in the evaluation of tumor response to transarterial treatments has been investigated. Differently from the good sensitivity shown with cholangiocarcinoma and hepatic metastases of colorectal cancer treated with transarterial chemoembolization (TACE), PET has shown to be of little diagnostic value with respect to injected MDCT and MRI for HCC in intermediate stage treated with TACE. Only under specific circumstances, as in the case of strong PET positivity found in pre-treatment evaluation or the presence of a large intrahepatic tumor burden treated with yttrium90-radioembolization has PET shown accuracy in early evaluation of tumor response ${ }^{[39]}$.

In conclusion, even if 18 F-FDG-PET does not acquire a definite role in guidelines due to its low sensibility in revealing HCC, it has proved useful in specific instances, such as prior to listing patients with large HCC for liver transplantation, before major resections or when there is suspicion of an extra-hepatic neoplastic diffusion. In the last EASL guidelines FDG PET-scan is not recommended for early diagnosis of HCC because of the high rate of false negative cases but uptake on 18F-FDG-PET seems to be of potential prognostic value. Therefore, it may facilitate the selection of patients for surgical resection or liver transplantation $^{[28]}$. 


\section{HCC IN NON-CIRRHOTIC PATIENTS}

HCC occurring in non-cirrhotic livers is uncommon and the clinical presentation is very different from that observed in cirrhosis. Since the tumor occurs in subjects not known to be at risk of HCC, the diagnosis is generally delayed and therefore the tumor is larger than commonly seen in cirrhotic patients. The problem is whether the radiological characteristics that are decisive for the diagnosis of HCC on cirrhosis can be translated in this different clinical situation. Di Martino et al. ${ }^{[40]}$ retrospectively reviewed histopathological and laboratory findings of 30 non-cirrhotic patients with 32 HCCs. MDCT and gadobenate dimeglumine enhanced MRI were evaluated. Imaging patterns were compared directly with HCC findings in a matched group of cirrhotic patients. The imaging appearance at MDCT and contrast-enhanced MRI was typical in $27(84.3 \%)$ and $28(87.5 \%)$ cases, respectively. Most lesions presented as a well-differentiated large solitary mass, with well-defined margins, areas of necrosis and peripheral capsule. No significant differences in HCC pattern were observed between cirrhotic and non-cirrhotic liver. But in the last EASL guidelines in noncirrhotic liver, imaging alone is not considered sufficient and histological assessment is required to establish the diagnosis of HCC and has the additional advantage of providing further information regarding the nontumourous liver tissue ${ }^{[28]}$.

\section{MODIFIED RESPONSE EVALUATION CRITERIA IN SOLID TUMORS FOR THE EVALUATION OF HCC RESPONSE TO TREATMENTS}

Radiology plays an important role not only for the diagnosis but also for the evaluation of the response both to locoregional and to systemic treatments of HCC. Until 2010, EASL and AASLD have recommended Response Evaluation Criteria In Solid Tumors (RECIST) and WHO criteria in their guidelines for the management of HCC for the evaluation of response to treatment. The application of these criteria requires the measurement of the major diameter of the HCC nodule/s. However, because of the relevance of the necrotic portion of a treated nodule with respect to its global size, a new version of RECIST modified for HCC, formally taking into account only the vital tissue in each HCC lesion, was published in $2010^{[41]}$. These criteria have been endorsed in 2012 by EASL and European Organisation for Research and Treatment of Cancer (EORTC) in their guidelines and are currently still the gold standard for the assessment of radiological response, confirmed in the last version of EASL guidelines ${ }^{[4,28]}$. The application of modifiedRECIST (mRECIST) requires taking into account those HCC nodules which are clearly visible, measurable and showing the typical "hallmark of wash-in and wash-out" as "target lesions", while all the HCC localizations not definitively measurable or with an atypical post-contrast appearance such as intrahepatic lesions which show infiltrative behavior with poorly defined margins and poorly defined hyperenhancement, malignant thrombosis, neoplastic ascites, adenopathies, very small and/or numerous diffuse lesions, are to be considered "non target lesions". According to mRECIST, only "target lesions" can undergo a dimensional evaluation of their vital portion (tissue showing arterial hyperenhancement and venous/delayed washout) by the measurement of its longest diameter. The response assessment is to be based on the comparison of this size before and after the treatment. On the contrary, "non target lesions" can be monitored over time on the basis of their absence/presence (i.e., neoplastic ascites) or of their measurement according to RECIST (longest diameter of the lesion as a whole). With respect to "target lesions", the possible responses are: a complete response (CR) defined by the disappearance of all arterial hyperenhancement in all target lesions; a partial response (PR) in the case of a reduction of at least $30 \%$ in the sum of the diameters of the vital portions of the target lesions; progressive disease (PD) if an increase of at least $20 \%$ in the sum of the longest diameters of all target lesions is observed; and stable disease (SD) if neither PR nor PD definition criteria can be satisfied [Table 2].

With respect to "non-target lesions": a CR will correspond to the disappearance of all enhancing tissue in all of them, an incomplete response/SD is defined by the persistence of vital tissue in at least one "non target lesion"; a PD is defined by the appearance of a new lesion or the unequivocal worsening of at least one of the 
Table 2. Comparison between Response Evaluation Criteria In Solid Tumors and Modified Response Evaluation Criteria In Solid Tumors for target lesions ${ }^{[4]]}$

\begin{tabular}{|c|c|}
\hline RECIST & mRECIST for HCC \\
\hline CR = Disappearance of all target lesions & $\begin{array}{l}\text { CR = Disappearance of any intratumoral arterial enhancement in all } \\
\text { target lesions }\end{array}$ \\
\hline $\begin{array}{l}\text { PR = At least a } 30 \% \text { decrease in the sum of diameters of all target } \\
\text { lesions, taking as reference the baseline sum of the diameters of } \\
\text { target lesions }\end{array}$ & $\begin{array}{l}\text { PR }=\text { At least } 30 \% \text { decrease in the sum of diameters of viable } \\
\text { (enhancement in the arterial phase) target lesions, taking as } \\
\text { reference the baseline sum of the diameters of target lesions }\end{array}$ \\
\hline $\begin{array}{l}\text { SD }=\text { Any cases that do not qualify for either partial response or } \\
\text { progressive disease }\end{array}$ & $\begin{array}{l}\mathrm{SD}=\text { Any cases that do not qualify for either partial response or } \\
\text { progressive disease }\end{array}$ \\
\hline $\begin{array}{l}\mathrm{PD}=\mathrm{An} \text { increase in } 20 \% \text { in the sum of diameters of target lesions, } \\
\text { taking as reference the smallest sum of the diameters of target } \\
\text { lesions recorded since treatment started }\end{array}$ & $\begin{array}{l}\mathrm{PD}=\mathrm{An} \text { increase in } 20 \% \text { in the sum of diameters of viable } \\
\text { (enhancing) target lesions, taking as reference the smallest sum of } \\
\text { the diameters of target lesions recorded since treatment started }\end{array}$ \\
\hline
\end{tabular}

RECIST: Response Evaluation Criteria In Solid Tumors; mRECIST: Modified Response Evaluation Criteria In Solid Tumors; HCC: hepatocellular carcinoma; CR: complete response; PR: partial response; SD: stable disease; PD: progressive disease

known "non target lesions". After having assigned a definition for each category of lesions (target/non target) it will be possible to define the "overall response according to mRECIST" by cross-referencing each response obtained in the two categories of lesions as shown in Table 3.

Subsequent publications have reported a good correlation between the objective response evaluated with mRECIST and overall survival, both after the application of loco-regional treatments ${ }^{[42]}$ and also after systemic therapies ${ }^{[43]}$. However, the application of mRECIST criteria in clinical practice is sometimes challenging due to their complexity and also because it is not rare to find an HCC represented exclusively by "non target lesions" or by nodular hepatic lesions which do not exhibit the traditional post-contrast "HCC hallmark". As such, their use is contraindicated. Finally, it remains to be established that mRECIST is superior to RECIST and a comparative evaluation is therefore required ${ }^{[44]}$. A recent analysis of two phase II trials in patients treated with sorafenib or nintedanib showed that both RECIST and mRECIST response were correlated with overall survival, with similar discriminative abilities in multivariate analysis ${ }^{[45]}$. It was also shown that mRECIST and RECIST are equivalent in the evaluation of progression, which is the most important endpoint in regards to therapeutic decisions.

\section{IN THE FUTURE}

The development of artificial intelligence will probably allow in the future to improve the interpretation of images obtained with the ultrasound, MDTC or MRI with contrast medium limiting diagnostic errors computer-aided diagnosis (CAD) is one of the most important research topics in radiology and medicine. A software based on the recognition of the contrast features of the lesions calculates the probability that they are benign or malignant. Moga et al. ${ }^{[46]}$ developed a CAD prototype used to analyze 97 videos of good quality CEUS [34\% HCC, $12.3 \%$ hypervascular metastases, $11.3 \%$ hypovascular metastases, $24.7 \%$ of hemangiomas, $17.5 \%$ of focal nodular hyperplasia (FNH)]. The authors evaluated the diagnostic performance of two young doctors, two experts and CAD in the diagnosis of benign vs. malignant lesions. The CAD was useful in improving the diagnostic skills of young doctors, especially when integrated with clinical data, but was lower than the skills of experienced doctors. The most frequently misdiagnosed lesions were FNH and $\mathrm{HCC}^{[46]}$.

Kim et al ${ }^{[47]}$ developed and evaluated a CAD program for hepatic lesions on MRI for the classification of HCC risk according to the LI-RADS criteria. MRI images of the livers of 41 patients with hyperenhancing liver lesions classified as LR 3, 4 and 5 were evaluated by two radiologists. The agreement on the classification of lesions by radiologists and CAD was $76 \%-83 \%$, while the agreement between radiologists was $78 \%$. 
Table 3. Overall response according to Modified Response Evaluation Criteria In Solid Tumors for hepatocellular carcinoma

\begin{tabular}{lccc}
\hline Target lesions & Nontarget lesions & New lesions & Overall response \\
\hline CR & CR & No & CR \\
CR & IR/SD & No & PR \\
PR & Non-PD & No & PR \\
SD & Non-PD & No & SD \\
PD & Any & Yes or no & PD \\
Any & PD & Yes or no & PD \\
Any & Any & Yes & PD \\
\hline
\end{tabular}

CR: complete response; PR: partial response; SD: stable disease; PD: progressive disease; IR: incomplete response

\section{DECLARATIONS}

\section{Authors' contributions}

Conception of the work,drafting and revisingthe work: de Santis A, Gallusi G

\section{Availability of data and materials}

Not applicable.

\section{Financial support and sponsorship}

None.

\section{Conflicts of interest}

All authors declared that there are no conflicts of interest.

\section{Ethical approval and consent to participate}

Not applicable.

\section{Consent for publication}

Not applicable.

\section{Copyright}

(C) The Author(s) 2019.

\section{REFERENCES}

1. Bréchot C, Jaffredo F, Lagorce D, Gerken G, Meyer zum Büschenfelde K, et al. Impact of HBV, HCV and GBV-C/HGV on hepatocellular carcinomas in Europe: results of a European concerted action. J Hepatol 1998;29:173-83.

2. Smith R. Screening fundamentals. J Natl Cancer Inst Monogr 1997;(22):15-9.

3. Bruix J, Sherman M; American Association for the Study of Liver Diseases. Management of hepatocellular carcinoma: an update. Hepatology 2011;53:1020-2.

4. European Association For The Study Of The Liver; European Organisation For Research And Treatment Of Cancer. EASL-EORTC clinical practice guidelines: management of hepatocellular carcinoma. J Hepatol 2012;56:908-43.

5. Heimbach JK, Kulik LM, Finn RS, Sirlin CB, Abecassis MM, et al. AASLD guidelines for the treatment of hepatocellular carcinoma. Hepatology 2018;67:358-80.

6. Bennett GL, Krinsky GA, Abitbol RJ, Kim SY, Theise ND, et al. Sonographic detection of hepatocellular carcinoma and dysplastic nodules in cirrhosis: correlation of pretransplantation sonography and liver explant pathology in 200 patients. AJR Am J Roentgenol 2002;179:75-80

7. Bolondi L. Screening for hepatocellular carcinoma in cirrhosis. J Hepatol 2003;39:1076-84.

8. Nowicki TK, Markiet K, Szurowska E. Diagnostic imaging of hepatocellular carcinoma - a pictorial essay. Curr Med Imaging Rev 2017;13:140-53.

9. Kudo M, Izumi N, Kokudo N, Matsui O, Sakamoto M, et al. Management of hepatocellular carcinoma in Japan: consensus-based clinical practice guidelines proposed by the Japan Society of Hepatology (JSH) 2010 updated version. Dig Dis 2011;29:339-64.

10. Omata M, Lesmana LA, Tateishi R, Chen PJ, Lin SM, et al. Asian Pacific Association for the Study of the Liver consensus recommendations on hepatocellular carcinoma. Hepatol Int 2010;4:439-74.

11. Russo FP, Imondi A, Lynch EN, Farinati F. When and how should we perform a biopsy for HCC in patients with liver cirrhosis in 
2018? A review. Dig Liver Dis 2018;50:640-6.

12. Ryu SW, Bok GH, Jang JY, Jeong SW, Ham NS, et al. Clinically useful diagnostic tool of contrast enhanced ultrasonography for focal liver masses: comparison to computed tomography and magnetic resonance imaging. Gut Liver2014;8:292-7.

13. Dulku G, Dhillon R, Goodwin M, Cheng W, Kontorinis N, et al. The role of imaging in the surveillance and diagnosis of hepatocellular cancer. J Med Imaging Radiat Oncol 2017;61:171-9.

14. Ayuso C, Rimola J, Vilana R, Burrel M, Darnell A, et al. Diagnosis and staging of hepatocellular carcinoma (HCC): current guidelines. Eur J Radiol 2018;101:72-81.

15. Bruix J, Sherman M; Practice Guidelines Committee, American Association for the Study of Liver Diseases. Management of hepatocellular carcinoma.Hepatology 2005;42:1208-36.

16. Piana G, Trinquart L, Meskine N, Barrau V, Beers BV, et al. New MR imaging criteria with a diffusion-weighted sequence for the diagnosis of hepatocellular carcinoma in chronic liver diseases. J Hepatol 2011;55:126-32.

17. Raman SS, Leary C, Bluemke DA, Amendola M, Sahani D, et al. Improved characterization of focal liver lesions with liver-specific gadoxetic acid disodium-enhanced magnetic resonance imaging: a multicenter phase 3 clinical trial. J Comput Assist Tomogr 2010;34:163-72

18. Tsuboyama T, Onishi H, Kim T, Akita H, Hori M, et al. Hepatocellular carcinoma: hepatocyte-selective enhancement at gadoxetic acid-enhanced MR imaging--correlation with expression of sinusoidal and canalicular transporters and bile accumulation. Radiology 2010;255:824-33.

19. Ichikawa T, Saito K, Yoshioka N, Tanimoto A, Gokan T, et al. Detection and characterization of focal liver lesions: a Japanese phase III, multicenter comparison between gadoxetic acid disodium-enhanced magnetic resonance imaging and contrast-enhanced computed tomography predominantly in patients with hepatocellular carcinoma and chronic liver disease. Invest Radiol 2010;45:133-41.

20. Roberts LR, Sirlin CB, Zaiem F, Almasri J, Prokop LJ, et al. Imaging for the diagnosis of hepatocellular carcinoma: a systematic review and meta-analysis. Hepatology 2018;67:401-21.

21. ACR. Liver reporting and data system. Available from: https://www.acr.org/Clinical-Resources/Reporting-and-Data-Systems/LIRADS/LI-RADS1. [Last accessed on 20 Dec 2018]

22. ACR. CT/MRI LI-RADS ${ }^{\circledR}$ v2017. Available from: https://www.acr.org/Clinical-Resources/Reporting-and-Data-Systems/LI-RADS/ CT-MRI-LI-RADS-v2017. [Last accessed on 20 Dec 2018]

23. ACR. Ultrasound LI-RADS ${ }^{\circledR}$ v2017. Available from: https://www.acr.org/Clinical-Resources/Reporting-and-Data-Systems/LI-RADS/ Ultrasound-LI-RADS-v2017. [Last accessed on 20 Dec 2018]

24. Wildner D, Bernatik T, Greis C, Seitz K, Neurath MF, et al. CEUS in hepatocellular carcinoma and intrahepatic cholangiocellular carcinoma in 320 patients - early or late washout matters: a subanalysis of the DEGUM multicenter trial. Ultraschall Med 2015;36:132-9.

25. ACR. CEUS LI-RADS ${ }^{\circledR}$ v2017. Available from: https://www.acr.org/Clinical-Resources/Reporting-and-Data-Systems/LI-RADS/ CEUS-LI-RADS-v2017. [Last accessed on 20 Dec 2018]

26. Wilson SR, Lyshchik A, Piscaglia F, Cosgrove D, Jang HJ, et al. CEUS LI-RADS: algorithm, implementation, and key differences from CT/MRI. Abdom Radiol (NY) 2018;43:127-42.

27. Terzi E, Iavarone M, Pompili M, Veronese L, Cabibbo G, et al. Contrast ultrasound LI-RADS LR-5 identifies hepatocellular carcinoma in cirrhosis in a multicenter restropective study of 1,006 nodules. J Hepatol 2018;68:485-92.

28. Galle PR, Forner A, Llovet JM, Mazzaferro V, Piscaglia F, et al. EASL clinical practice guidelines: management of hepatocellular carcinoma. J Hepatol 2018;69:182-236.

29. Kojiro M, Wanless IR, Alves V, Badve S, Balabaud C, et al. Pathologic diagnosis of early hepatocellular carcinoma: a report of the international consensus group for hepatocellular neoplasia. Hepatology 2009;49:658-64.

30. Yoon SH, Lee JM, So YH, Hong SH, Kim SJ, et al. Multiphasic MDCT enhancement pattern of hepatocellular carcinoma smaller than $3 \mathrm{~cm}$ in diameter: tumor size and cellular differentiation. AJR Am J Roentgenol 2009;193:W482-9.

31. Park YS, Lee CH, Kim JW, Shin S, Park CM. Differentiation of hepatocellular carcinoma from its various mimickers in liver magnetic resonance imaging: what are the tips when using hepatocyte-specific agents? World J Gastroenterol 2016;22:284-99.

32. Kitao A, Matsui O, Yoneda N, Kozaka K, Shinmura R, et al. The uptake transporter OATP8 expression decreases during multistep hepatocarcinogenesis: correlation with gadoxetic acid enhanced MR imaging. Eur Radiol 2011;21:2056-66.

33. Sirlin CB, Kielar AZ, Tang A, Bashir MR. LI-RADS: a glimpse into the future. Abdom Radiol (NY) 2018;43:231-6.

34. Park JW, Kim JH, Kim SK, Kang KW, Park KW, et al. A prospective evaluation of 18F-FDG and 11C-acetate PET/CT for detection of primary and metastatic hepatocellular carcinoma. J Nucl Med 2008;49:1912-21.

35. Park JH, Kim DH, Kim SH, Kim MY, Baik SK, et al. The clinical implications of liver resection margin size in patients with hepatocellular carcinoma in terms of positron emission tomography positivity. World J Surg 2018;42:1514-22.

36. Kornberg A, Küpper B, Tannapfel A, Büchler P, Krause B, et al. Patients with non-[18 F]fludeoxyglucose-avid advanced hepatocellular carcinoma on clinical staging may achieve long-term recurrence-free survival after liver transplantation. Liver Transpl 2012;18:53-61.

37. Ijichi H, Shirabe K, Taketomi A, Yoshizumi T, Ikegami T, et al. Clinical usefulness of (18) F-fluorodeoxyglucose positron emission tomography/computed tomography for patients with primary liver cancer with special reference to rare histological types, hepatocellular carcinoma with sarcomatous change and combined hepatocellular and cholangiocarcinoma. Hepatol Res 2013;43:481-7.

38. Yoh T, Seo S, Ogiso S, Kawai T, Okuda Y, et al. Proposal of a new preoperative prognostic model for solitary hepatocellular carcinoma incorporating 18F-FDG-PET imaging with the ALBI grade. Ann Surg Oncol 2018;25:542-9.

39. Hartenbach M, Weber S, Albert NL, Hartenbach S, Hirtl A, et al. Evaluating treatment response of radioembolization in intermediatestage hepatocellular carcinoma patients using 18F-fluoroethylcholine PET/CT. J Nucl Med 2015;56:1661-6.

40. Di Martino M, Saba L, Bosco S, Rossi M, Miles KA, et al. Hepatocellular carcinoma (HCC) in non-cirrhotic liver: clinical, radiological and pathological findings. Eur Radiol 2014;24:1446-54. 
41. Lencioni R, Llovet JM. Modified RECIST (mRECIST) assessment for hepatocellular carcinoma. Semin Liver Dis 2010;30:52-60.

42. Shim JH, Lee HC, Kim SO, Shin YM, Kim KM, et al. Which response criteria best help predict survival of patients with hepatocellular carcinoma following chemoembolization? A validation study of old and new models. Radiology 2012;262:708-18.

43. Lencioni R, Montal R, Torres F, Park JW, Decaens T, et al. Objective response by mRECIST as a predictor and potential surrogate end-point of overall survival in advanced HCC. J Hepatol 2017;66:1166-72.

44. Edeline J, Palmer D, Blanc JF, Campillo-Gimenez B, Merle P, et al. mRECIST for systemic therapies: more evidence is required before recommendations can be made. J Hepatol 2017;67:195.

45. Meyer T, Palmer DH, Cheng AL, Hocke J, Loembé AB, et al. MRECIST to predict survival in advanced hepatocellular carcinoma: analysis of two randomised phase II trials comparing nintedanib vs sorafenib. Liver Int 2017;37:1047-55.

46. Moga TV, Popescu A, Sporea I, Danila M, David C, et al. Is contrast enhanced ultrasonography a useful tool in a beginner's hand? How much can a computer assisted diagnosis prototype help in characterizing the malignancy of focal liver lesions? Med Ultrason 2017; 19:252-8.

47. Kim Y, Furlan A, Borhani AA, Bae KT. Computer-aided diagnosis program for classifying the risk of hepatocellular carcinoma on MR images following liver imaging reporting and data system (LI-RADS). J Magn Reson Imaging 2018;47:710-22. 